\title{
SULFADIAZINE RESISTANT STREPTOCOCCAL INFECTIONS IN A CIVILIAN COMMUNITY 1,2
}

\author{
By ROSWELL D. JOHNSON AND THOMAS L. HARTMAN \\ (From the Mary Imogene Bassett Hospital, Cooperstowen, New York and the U. S. Naval \\ Research Unit at the Hospital of The Rockefeller Institute for Medical Research \\ and the Hospital of The Rockefeller Institute for Medical Research)
}

(Received for publication November 13, 1946)

During a program of mass chemoprophylaxis of streptococcal infections in the Armed Forces (1), it was noted that the effectiveness of such a regimen was lost with the appearance of sulfonamide-resistant strains of hemolytic streptococci. The occurrence and spread of these resistant strains in susceptible populations in the U. S. Army and Navy have been reported $(2,3$, 4 ), and have been the subject of considerable speculation and theoretical implications. To date there have been no reports as to the incidence of streptococcal epidemics in the civilian population due to sulfonamide-resistant strains. It is the purpose of this paper to report the occurrence of such an epidemic in a civilian community due to a sulfadiazine-resistant group A, type 19 hemolytic streptococcus.

\section{METHODS OF STUDY}

Early in 1946 a small epidemic of scarlet fever occurred in Cooperstown, New York. Since the first 8 patients with scarlet fever were found to harbor type 19 hemolytic streptococci in their nasopharynges, it was decided to determine whether we were dealing with sulfonamideresistant strains. All 8 strains of hemolytic streptococci were found to be resistant to the action of sodium sulfadiazine in a concentration of $25 \mathrm{mgm}$. per cent.

Because of the unique opportunity to study the incidence of sulfonamide-resistant hemolytic streptococci in a small community, it was decided to test the sulfonamide sensitivity of all hemolytic streptococcus cultures sent to the laboratory in this area. Cultures were obtained from patients, living in Cooperstown, New York, and the sur-

1 Assisted in part by a contract between The Rockefeller Institute for Medical Research and the Commission on Hemolytic Streptococcal Infections, Board for the Investigation and Control of Influenza and Other Epidemic Diseases in the Army, Preventive Medicine Service, Office of the Surgeon General, United States Army.

2 This article has been released for publication by the Division of Publications of the Bureau of Medicine and Surgery of the U. S. Navy. The opinions and views set forth in this article are those of the writers and are not to be considered as reflecting the policies of the Navy Department. rounding area of Otsego County, who were suspected of having streptococcal infections. Records were kept as to the source of the cultures and the clinical diagnosis. No attempt was made to do a widespread survey on a healthy group to determine carrier rates.

All culture swabs were sent to the Otsego County Laboratory at the Mary Imogene Bassett Hospital or to a nearby branch laboratory. The swabs were streaked on 5 per cent rabbit blood agar which was incubated for 24 hours at $37^{\circ} \mathrm{C}$. Single colonies of beta hemolytic streptococci were transferred to Avery's media on the following day and incubated for 6 hours. A loopful of this growth was then streaked to a blood agar plate, checked for purity, and the group determined. The group A hemolytic streptococci isolated were grouped and typed at the Rockefeller Institute Hospital by the precipitin technique (5), and the sulfonamide sensitivity was determined by the method of Wilson (6).

\section{EPIDEMIOLOGICAL SITUATION AND RESULTS}

From February 15 to May 1, 1946, streptococcal infections were moderately prevalent in Cooperstown, New York. During this period 100 patients with varied group A hemolytic streptococcal in-

TABLE I

Type distribution and sulfadiazine resistance of group $A$ hemolytic streptococci

\begin{tabular}{c|c|c}
\hline \hline Infecting type & No. of cases & $\begin{array}{c}\text { No. of sulfadiazine- } \\
\text { resistant strains }\end{array}$ \\
\hline 1 & 1 & \\
2 & 2 & \\
3 & 28 & \\
4 & 2 & \\
5 & 2 & \\
6 & 1 & \\
9 & 2 & \\
12 & 1 & \\
13 & 2 & \\
14 & 1 & \\
17 & 4 & \\
28 & 29 & \\
NC & 24 & $23^{2}$ \\
19 & & \\
\hline
\end{tabular}

$1 \mathrm{NC}$ indicates strains not classified by the precipitin method with available diagnostic sera.

2 These 23 strains all resisted $25 \mathrm{mgm}$. per cent of sodium sulfadiazine in the media. 


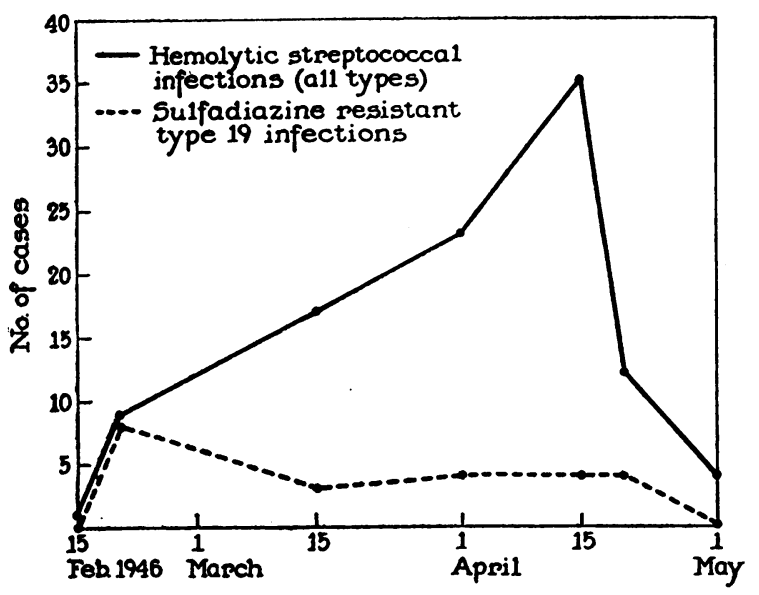

FIG. 1

fections were cultured, with the type distribution indicated in Table I. The cultures were obtained from patients with scarlet fever, pharyngitis, otitis media, wound infections, blood stream infections, and pneumonia. The time incidence of cases during this period is shown in Chart 1. By May 1,1946 , the incidence of streptococcal infections had decreased to the point where it was no longer considered of value to continue the study.

The most common typable streptococcus was type 3, which was responsible for 28 cases of hemolytic streptococcal infections. Type 19 was the cause of 24 cases of disease, with 23 of these strains being resistant to $25 \mathrm{mgm}$. per cent sodium sulfadiazine and 1 strain non-resistant. Nineteen cases were due to the other types listed in Table I, while 29 were caused by untypable strains. Only type 19 hemolytic streptococci were resistant to the action of sodium sulfadiazine.

Since our main interest in this report is concerned with the occurrence of type 19 sulfonamideresistant hemolytic streptococci, the epidemiological situation in respect to these infections will be discussed briefly. Of the 23 persons infected with this sulfonamide resistant strain, 14 were under 15 years of age, 10 of public school age, and 4 of pre-school age, 9 cases occurred in adults.

The first case of scarlet fever in this study developed on February 15, 1946, in a 9-year-old boy who attended the public school. Following the onset of this patient's illness, several classmates developed scarlet fever, and from their nasopharynges type 19 sulfadiazine-resistant streptococci were isolated. The next cases developing due to resistant type 19 strains appeared in parents, sibblings, or contacts of the children originally infected in the public school. However, 3 patients were from widely separated communities and had no known contact with any of the previously mentioned cases. None had any known contact with recently discharged or active members of the Armed Forces.

Of these cases of drug-resistant infection, 14 were clinical scarlet fever, and 9 were merely pharyngitis. One case of scarlet fever and one case of pharyngitis were complicated by otitis media and one patient developed rheumatic fever following pharyngitis due to this type 19 sulfadiazine-resistant hemolytic streptococcus. None of these cases presented any special therapeutic problems, nor were they clinically different from infections due to other types of hemolytic streptococci. The incidence of infection with this particular organism was rather evenly distributed throughout the period of this study with the peak occurring early (see Figure 1).

\section{COM MENT}

The possibility of a spread of sulfadiazine-resistant hemolytic streptococci to the civilian population has been anticipated ever since their appearance' in the Armed Forces $(2,3,4)$. The present report indicates that such resistant strains have, indeed, been responsible for infections in at least one civilian community and that similar occurrences may complicate future problems of therapy among civilians.

The mode of development of resistant strains of bacteria is still a matter of discussion. It has been demonstrated that strains of streptococci with low or medium degrees of sulfadiazine resistance appeared in the Army (7) about the time that the program of mass sulfa-prophylaxis had been in progress in the Navy (1) for several months. It had been suggested that these slightly resistant strains were mutants that might possibly have been precursors of the more highly resistant strains later established. The possibility cannot, however, be eliminated that there was a transfer of resistant strains to soldiers from certain Naval personnel, who were subjects of the mass prophylaxis program, and hence possible carriers of highly resistant streptococci. It is noteworthy that no evidence of sulfonamide resistance was 
found in any strains of hemolytic streptococci prior to the institution of the mass chemoprophylaxis programs $(2,8)$.

The possibility that sulfonamide-resistant hemolytic streptococci could arise in the civilian population must be considered. The evidence to date, however, would seem to indicate that all strains of hemolytic streptococci which were present in the civilian population prior to the time of mass chemoprophylaxis in the Armed Forces were susceptible to the action of the sulfonamides. Evidence (8) has also been presented to show that the usual therapeutic doses of sulfonamides, such as might be prescribed in a civilian population, did not cause the development of resistant organisms.

Since the sulfadiazine-resistant hemolytic streptococcal infections reported in this study were caused by a type 19 strain, which was known to be a prominent resistant strain in the Armed Forces, it seems probable that this strain was introduced into this civilian community by military personnel even though a history of recent contact with sick soldiers and sailors could not be elicited. Contact of civilians with discharged veterans, some of whom might have been healthy carriers of these resistant strains, could hardly have been avoided. The fact that most cases of sulfonamide-resistant infections here reported occurred among school children is noteworthy, since all previous reports of sulfonamide-resistant infections occurred in adults.

Although the cases mentioned in this report did not offer any problems as to therapy, one cannot overlook the importance of determining whether one is dealing with an organism sensitive to the chemotherapeutic agent being administered. Since the clinical picture of infections caused by resistant strains of hemolytic streptococci is no different than those caused by non-resistant strains, one can only determine this property by isolation and testing of the organism causing the infection. From an epidemiological and clinical point of view, the determination of the presence of resistant strains would be of definite value if the prevention and treatment of resistant-strain complications is to be effectively accomplished.

The appearance of increasing numbers of bacteria which are resistant to various chemothera- peutic agents, formerly effective against them, suggests the desirability of a careful bacteriological diagnosis of infectious agents prior to treatment with specific antibacterial drugs, especially when there is an obvious failure of clinical response to the chemotherapeutic agent under consideration.

\section{SUM MARY}

Twenty-three cases of type 19 sulfadiazine-resistant hemolytic streptococcus infections occurred in a civilian community. None of the cases presented any problem as to treatment nor were there any serious complications. Attention is directed to the importance of determining the sensitivity of infecting bacteria to chemotherapeutic agents prior to the start of treatment with specific agents.

The authors wish to thank Dr. Ann Kuttner and Dr. Homer F. Swift for their interest and criticism during this study.

Dr. Walter Eells, Walton, New York, and Dr. George Rosenthal, Cherry Valley, New York, were instrumental in obtaining many of the streptococcal cultures used in this study. Valuable technical assistance was rendered by Mrs. Winifred Cary, Cooperstown, New York, and Miss Jeanne Epstein of The Rockefeller Institute Hospital, New York, New York.

\section{BIBLIOGRAPHY}

1. Coburn, A. F., Mass sulfadiazine prophylaxis of respiratory diseases in the U. S. Navy. Bull. New York Acad. Med., 1945, 21, 281.

2. Sulfadiazine resistant strains of beta hemolytic streptococci : Appearance during the course of sulfadiazine prophylaxis at a large naval training center, Epidemiology Unit Number 22. J. A. M. A., 1945, 129, 921.

3. Damrosch, D. S., Chemoprophylaxis and sulfonamide resistant streptococci. J. A. M. A., 1946, 130, 124.

4. (a) Delameter, E. D., Jennings, R., and Wallace, A. W., Preliminary report of an outbreak of streptococcal disease caused by a sulfadiazine resistant group A, type 17 hemolytic streptococcus. J. Infect. Dis., 1946, 78, 118.

(b) Mitchell, R. B., et al., The interpost dissemination of epidemic strains of hemolytic streptococci by troop movements. J. Infect. Dis., 1946, 78, 128.

(c) Roberg, N. B., An epidemic caused by a sulfadiazine resistant strain of the streptococcus hemolyticus (group A, type 17). J. Infect. Dis., 1946, 78, 135.

(d) Wilson, O. G., An outbreak of sulfadiazine resistant streptococcus infection at Lowry Field, Colorado. J. Infect. Dis., 1946, 78, 147. 
5. Swift, H. F., Wilson, A. T., and Lancefield, R. C. Typing group A hemolytic streptococci by $M$ precipitin reactions in capillary pipettes. J. Exper. Med., 1943, 78, 127.

6. Wilson, A. T., Method for testing in vitro resistance of group A hemolytic streptococci to sulfonamides. Proc. Soc. Exper. Biol. \& Med., 1945, 58, 130.
7. Rantz, L. A., Randall, E., Spink, W. W., and Boisvert, P. J., Sulfonamide and penicillin resistance of group A hemolytic streptococci. Proc. Soc. Exper. Biol. \& Med., 1946, 62, 54.

8. Hartman, T. L., Sulfonamide sensitivity determinations of hemolytic streptococci isolated from patients before and after treatment with sulfadiazine. Bull. Johns Hopkins Hosp., 1946, 79, 342. 